Ivan Ezquerra-Romano* and Angel Ezquerra*

\title{
Highway to thermosensation: a traced review, from the proteins to the brain
}

DOI 10.1515/revneuro-2016-0039

Received June 30, 2016; accepted August 7, 2016; previously published online October 18, 2016

\begin{abstract}
Temperature maintenance and detection are essential for the survival and perpetuation of any species. This review is focused on thermosensation; thus a detailed and traced explanation of the anatomical and physiological characteristics of each component of this sensation is given. First, the proteins that react to temperature changes are identified; next, the nature of the neurons involved in thermosensation is described; and then, the pathways from the skin through the spinal cord to the brain are outlined. Finally, the areas of the brain and their interconnections where thermoperception arises are explained. Transduction of the external and internal temperature information is essentially mediated by the transient receptor potential ion channels (TRPs). These proteins are embedded in the neurons' membrane and they hyper- or de-polarize neurons in function of the intrinsic voltage and the temperature changes. There are distinct TRP sensors for different temperature ranges. Interestingly, the primary afferent neurons have either cold or hot receptors, so they are dedicated separately to cold or hot sensation. The information is transmitted by different pathways from the skin to the brain, where it either remains separated or is integrated to generate a response. It seems that both the determination of how thermoperception is produced and how we interact with the world are dependent on the particular arrangement and nature of the components, the way of transduction of information and the communication between these elements.
\end{abstract}

Keywords: labelled-line principle; temperature; thermoperception; thermoreceptors; transient receptor potential ion channels (TRPs).

\footnotetext{
*Corresponding authors: Ivan Ezquerra-Romano, Department of Cognitive, Perception and Brain Science, University College London, Gower Street, London WC1E 6BT, UK, e-mail: ivan.romano.14@ucl.ac.uk; and

Angel Ezquerra, Department of Didactics of Experimental Science, Faculty of Education, Complutense University of Madrid, C/Rector Royo Villanova, s/n, E-28040 Madrid, Spain, e-mail: angel.ezquerra@edu.ucm.es.

http://orcid.org/0000-0002-5736-9867
}

Introduction

Nature and evolution have provided humans with different tools to survive the mayhem of chemical and physical stimuli. We have a variety of chemoreceptors dispersed around the body that help us spot nutrition, noxious substances or a potential sexual partner. The most well-known and evolved chemical senses in humans are gustation and olfaction which detect the chemicals in the external environment. Olfaction detects volatile compounds and combines with gustation to improve the identification of foods. Other receptors are sensitive to physical changes in the environment. Vision, one of the most complex and important senses in the human body, detects electromagnetic radiation. Thus, half of the human cerebral cortex is considered to be dedicated to analysing visual information. The auditory system detects frequency and intensity of the periodic variations in air pressure, sound. Nearby, the vestibular system recognizes balance, movement and orientation of the body and head. All over the body, the skin and muscles also provide crucial information. There are different specialized receptors that are sensitive to touch (pressure), temperature (thermosensation), pain (noxious stimuli) and position (proprioception); the classification of theses senses is still unclear and there is no definitive consensus (Bear et al., 2007).

Key to the existence of life on Earth is the behaviour and nature of the variable temperature. The temperature (and pressure) established on Earth serves as a unique (from what is known) cultivation environment since many elements are stable and chemical reactions can take place at the right pace and in the correct amounts. In this wellestablished but fluctuating atmosphere, organisms need to be aware of and respond efficiently to temperature and its effect. There are many examples that show how creatures use thermal information in the everyday life for their survival: thermotropism in plants, thermal vision in snakes, sun basking in reptiles, seeking of shelter in mammals, etc. Most organisms need to stay within a particular temperature range in order to maintain their bodily functions; therefore, a temperature detection mechanism is needed.

A molecular explanation of the sense organs' function has not been available until recently. The elucidation of 
the details in the transduction processes has mainly been conducted in the last 40 years. This has helped to explain many aspects which have already allowed us to comprehend how the molecular and physiological characteristics contribute to the whole mechanism. However, the existing theories still need further improvements. A crucial step was taken when Cosens and Manning (1969) discovered the first transient receptor potential ion channels (TRPs) in mutant flies. After this, great advances have been made in the comprehension of the TRP channels. These channels have been found to be involved in many different processes, such as thermosensation.

This review is focused on thermosensation. Thus, a detailed and traced explanation of the anatomical and physiological characteristics of each component of this sensation is given. First, the proteins that react to temperature changes are identified; next, the nature of the neurons involved in thermosensation is described; and then, the pathways from the skin through the spinal cord to the brain are outlined. Finally, the areas of the brain and their interconnections where thermoperception arises are explained.

\section{Molecular level}

Transduction of the external and internal temperature is essentially mediated by some TRPs (Patapoutian et al., 2003). These proteins are embedded in the neurons' membrane and some of them are extremely sensitive to temperature changes. Thus, a change in temperature opens or closes these channels, and the internal and external concentrations of ions are consequently modified. In short, the cells are polarised in reaction to the temperature shifts so that a generator potential is established. In sensory neurons, when the generator potential shows enough size, spread or both, an action potential is generated. Accordingly, the initial change is transduced in a signal which can be transported.

Currently, 28 TRP channels have been found and these can be categorised into six families (Pedersen et al., 2005) which have different functions around the human's and other species' tissues. The families of the vanilloid TRP channels (TRPV), the melastatin TRP channels (TRPM) and the ankyrin transmembrane proteins channels (ANKTM) contain the proteins involved in thermosensation (Schepers and Ringkamp, 2010). Evidence that supports the special role of TRP channels in temperature sensation is their high $10^{\circ}$ temperature coefficient (Q10). This dimension states the rate of change of a chemical or biological reaction when temperature is increased by $10^{\circ} \mathrm{C}$. The rate of any reaction is affected by temperature; therefore any process in cells is affected by this variable. Most ion channels have a Q10 ranging between 1.5 and 2 (Hille, 2001; Xu et al., 2002; Benham et al., 2003), but thermoTRPs show Q10 values $>15$. For instance, TRPM8 has a Q10 of around 23.8 (Brauchi et al., 2004), TRPV3 has a Q10 of around 17-22, TRPV4 has a Q10 of 19.1 and TRPV1 has a Q10 of around 21 (Benham et al., 2003). Therefore, the sensitivity to temperature changes by TRP channels makes them candidates for becoming sensors of temperature.

The channels identified until now in the human body and associated with thermosensation can be classified into two groups: hot- and cold-sensitive. A neutral range between $30^{\circ} \mathrm{C}$ and $36^{\circ} \mathrm{C}$ has been defined. Below this range, cold stimuli are felt, whereas above it warm ones are experienced (Kenshalo et al., 1968; Lee et al., 1998; Lv and Liu, 2007). Within these two groups, we can distinguish between those that react to noxious and to innocuous temperature (Patapoutian et al., 2003). Noxious stimuli in the hot range have been defined to be over $\sim 43^{\circ} \mathrm{C}$ and under $\sim 17^{\circ} \mathrm{C}$ in the cold range (Davis and Pope, 2002; Patapoutian et al., 2003). Thanks to these receptors, humans can discriminate a temperature range on the skin from $<10^{\circ} \mathrm{C}$ to $>50^{\circ} \mathrm{C}$ (Voets et al., 2005).

It is noteworthy that these TRP channels are also expressed in the central nervous system and in other internal organs; this has led researchers to the idea that there is an endogenous agonist to these TRPs (Boulant and Dean, 1986; Boulant, 1998, 2000; Hori et al., 1999; Mezey et al., 2000; Guler et al., 2002). However, this review focuses on the sensors and their afferents which are involved in external temperature perception.

The scientific literature has always focused more on the hot-sensitive proteins; therefore, the understanding and information of these is much broader. The first TRP, TRPV1, was identified due to its response to capsaicin, the ingredient in red hot chilli peppers that elicits a burning pain sensation. TRPV1 activation occurs in the noxious spectrum, above $\sim 42^{\circ} \mathrm{C}$ (Caterina et al., 1997). The second channel discovered was TRPV2. It is activated by high noxious temperatures, having a threshold of $\sim 52^{\circ} \mathrm{C}$ (Caterina et al., 1999). The next hot receptor to be classified was TRPV3. The activation onset of this channel is around $33^{\circ} \mathrm{C}$, so it is the first one found to be discovered that transduces innocuous hot information, even though it still presents activity above the noxious limit $\left(\sim 43^{\circ} \mathrm{C}\right)$ (Smith et al., 2002; Xu et al., 2002). The last receptor found in the hot range was TRPV4. This is the only one that is not activated by noxious temperatures; its range of activation is from $\sim 27^{\circ} \mathrm{C}$ to $\sim 42^{\circ} \mathrm{C}$ (Guler et al., 2002). 
Within the cold-sensitive proteins, TRPM8 was the first cold receptor to be identified. This channel is sensitive to menthol which elicits a cool sensation when the receptor is exposed to this compound. Its activation range is between $\sim 28^{\circ} \mathrm{C}$ and $\sim 8^{\circ} \mathrm{C}$ (McKemy et al., 2002; Bautista et al., 2007). The last channel identified to be involved in thermosensation was TRPA1 (formerly ANKTM1). TRPA1 activates when temperatures fall below $\sim 17^{\circ} \mathrm{C}$ (Story et al., 2003). As aforementioned, a noxious stimulus is perceived below this temperature.

Furthermore, other studies have pinpointed other non-TRP proteins that seem to be involved in this process. Some examples, in the cold range, are TREK-1, a two-pore domain K+ channel (Maingret et al., 2000), DEGyENaC cation channel subunits (Askwith et al., 2001) and $\mathrm{Na} / \mathrm{K}$ ATPase (Carpenter, 1967; Pierau et al., 1974). In the hot range, P2X3, an ATP-gated cation selective ion channel, has been identified (Souslova et al., 2000).

In summary, the known thermosensitive mechanism collectively covers most of the temperature ranges to which we are sensitive. Thus, each TRP protein works at a particular temperature range and has its optimal point of activation within these ranges (Caterina et al., 1999; Nagy and Rang, 1999; Patapoutian et al., 2003; Voets et al., 2005). The physiological studies on the thermosensitive proteins have revealed a nonlinear relation between temperature increase/decrease and channel activity (Andrew and Craig, 2001; Xu et al., 2002).

Neurons associated with TRPV1, TRPV2 and TRPV3 increase their firing nonlinearly from their respective thresholds, probably until the cell and the proteins start to become damaged (Caterina et al., 1999; Andrew and Craig, 2001; Smith et al., 2002; Xu et al., 2002). Similarly, TRPA1 increases its activity nonlinearly when the temperature decreases to the noxious cold range. Cells with TRPV4 channels on their membrane show a positive nonlinear increase in firing within $\sim 27^{\circ} \mathrm{C}$ to $\sim 42^{\circ} \mathrm{C}$ and their peak of activity is $42^{\circ} \mathrm{C}$; after this point the current decreases. Similarly, TRPM8 neurons show an increase in activity within their range of activation $\left(\sim 8^{\circ} \mathrm{C}\right.$ to $\left.\sim 28^{\circ} \mathrm{C}\right)$ when the temperatures drop to its downside limit. These neurons show a decline in their activity when temperatures go down further than $\sim 8^{\circ} \mathrm{C}$; the peak current is again around this boundary (McKemy et al., 2002; Bautista et al., 2007). Consequently, we have the capacity to feel a continuous and interrupted increase or decrease from low to high temperatures. This is due to the overlapping activation and sensitivity of the existing TRPs, as well as a complex interaction of activation and inhibition of the neurons involved in this process.

It is noteworthy that chilli and menthol are not particularly sources of heat and cold, but the fact that TRPV1 and TRPM8, respectively, have binding sites for compounds in these substances increases the firing rate of the neurons coupled to these proteins. As a consequence, our brain 'tells' us we are feeling hot or cold, even though the skin temperature does not change.

The underlying mechanism that determines the opening and closing of the channels for a particular temperature is not completely known. Several different hypotheses have been proposed (Clapham, 2003). However, evidence that support the theories has just been found for two of them (Voets et al., 2005). The less verified theory states that changes in temperature produce channel-activating ligands. This mechanism has been just seen to work for TRPV4, but it has been rejected for TRPM8 and TRPV1, as they still work in cellfree patches (Watanabe et al., 2002; Brauchi et al., 2004; Voets et al., 2004, 2005). The most promising hypothesis for the mechanism of TRPs states that TRPM8, TRPV1 and TRPV3 are voltage-dependent gating channels, contrary to the first assumptions that identified thermoTRPs as voltage-independent (Brauchi et al., 2004; Voets et al., 2004; Chung et al., 2005).

The voltage-dependent model defines that temperature changes (cooling in the case of TRPM8 and heating for TRPV1) shift the channel activation curves to more physiological voltages. Two research works that elucidated this mechanism showed how temperature sensitivity is highly dependent on transmembrane voltage (Brauchi et al., 2004; Voets et al., 2004). Consequently, both temperature and voltage changes of the membrane are needed for increasing the probabilities of channel opening.

Unfortunately, the exact mechanisms that lead to the transduction of external thermal conditions to internal information are not fully understood. To fully comprehend these, we would need to know the structure of the gates and the process of their activation. Nevertheless, some insights have been achieved.

TRPs are non-selective cationic proteins, but have a high permeability to calcium ions. They are grouped into homo- or hetero-tetramers to form channels (Caterina et al., 1997; Clapham et al., 2001; Xu et al., 2002; Pedersen et al., 2005). Each individual protein is formed by six membrane-spanning domains which present a hydrophobic region between transmembrane domains 5 and 6 . Similar to all the transmembrane proteins, TRPs have an amino and a carboxy terminus; in this case both are cytoplasmic and the amino terminus has three ankyrin-repeat domains. TRPMs do not have these repeats (Caterina et al., 1997; Pedersen et al., 2005). The transmembrane domains 1-4 and the cytoplasmic terminal segments may contain the regulatory parts that control channel gating (Voets 
et al., 2005), whereas the hydrophobic region between domains 5 and 6 may be the ion passage.

On the other hand, some prominent advances about the structure of some voltage-gated ion channels (VGICs) regulatory domains and their functions have also been achieved (Jiang et al., 2002; Zagotta et al., 2003; Pioletti et al., 2006; Yuan et al., 2010). VGICs are outfitted with diverse cytoplasmic domains (Yu et al., 2005); such domains seem to provide meaning for chemical and physical stimuli that would influence opening and closing processes. Thus, several authors (Grandl et al., 2008; Bagriantsev et al., 2012) have suggested that these intracellular domains can serve as temperature sensors. In this regard, some VGIC superfamily members, such as TRPs (Brauchi et al., 2006; Clapham and Miller, 2011; Vriens et al., 2014), also seem to be gated by the temperature effect in their cytoplasmic domains. Contrarily, other researchers consider that the thermal response in VGIC superfamily members arises from elements distributed throughout the channel (Clapham and Miller, 2011; Chowdhury et al., 2014).

Strikingly, the VGIC superfamilies, including the bacterial voltage-gated sodium channels, share the architecture of the C-terminal cytoplasmic domain (CTD) (Figure 1). The CTD has two parts: the neck which is a membrane proximal four-helix bundle bearing a hydrophilic core (Shaya et al., 2014; Payandeh and Minor, 2015) and a C-terminal four-stranded coiled coil (Mio et al., 2010; Powl et al., 2010; Irie et al., 2012; Shaya et al., 2014; Payandeh and Minor, 2015). Some studies have proposed that the neck undergoes an order/disorder transition on channel opening (Shaya et al., 2014). Thus, the neck folds a stable structure versus a disordered one and this change depends on temperature. That is to say, the neck domain forms a metastable structure.

Arrigoni et al. (2016) have demonstrated that the neck has a temperature-dependent unfolding transition that is directly coupled to channel opening. The neck has two ion binding sites which have different activation energies and can shift its arrangement by the effect of temperature (Figure 1). This structure contributes to the neck rigidity and the CTD functional properties. Concretely, this domain is constrained on its $\mathrm{N}$ and $\mathrm{C}$ termini by the channel pore domain and a classic parallel four-stranded coiled coil, respectively. These physical constraints are influenced by the neck during the process of gating.

Furthermore, a comparison of the NaVAe1p and TRPA1 CTDs reveals striking structural similarities and it points to a unified mechanism in which the propensity of the neck region to adopt an order/disorder transition is the base to understand the voltage-dependent opening of

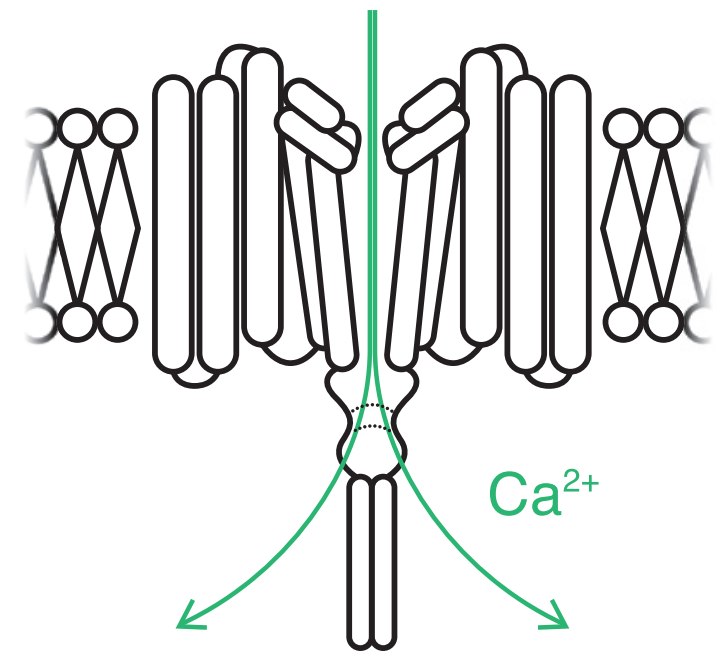

Figure 1: Representation of a thermoTRP in its open state. The dotted lines represent the hydrophilic core of the neck domain, which provides this structure with its metastable behaviour. Note that this is a theoretical diagram since the channels best described to date are bacterial sodium channels (Shaya et al., 2014; Arrigoni et al., 2016). Nevertheless, TRPs (with a high permeability to calcium ions) have been suggested to also rely on cytoplasmic elements for their temperature-dependent activity. Modified from Arrigoni et al. (2016), Shaya et al. (2014) and Voets et al. (2005).

the channel (Arrigoni et al., 2016). Obviously, these results establish that a discrete ion channel domain can serve as a temperature response element.

Another way of considering the mechanism of gating is in relation to the thermodynamical variables that govern the conformation of the molecular equilibrium. The enthalpy and the entropy changes are quite high for channel opening, resulting in a low Gibbs free energy (Brauchi et al., 2004; Nilius et al., 2005). Large enthalpy and entropy changes imply large structural rearrangements of the proteins. Since enthalpy and entropy are intrinsically dependent on temperature, these facts could mean that there exists a temperature-sensing domain in TRPM8 and TRPV1 (Brauchi et al., 2004). Why are TRPM8 and TRPV1 sensitive to cooling and heating, respectively? There is no consensus to the answer of this question. Voets et al. (2004) proposed a two-state model. They formulated that temperature sensitivity arises when there are differences in the activation energies associated with the opening and closing of the channel. Therefore, TRPM8 presents a higher closing activation energy and TRPV1 higher opening activation energy. On the other hand, Brauchi et al. (2004) proposed an allosteric model. In this model, the activation of the sensors to voltage and temperature and the channel opening compose three separate two-state equilibriums. 
Additionally, TRPs have been found to be regulated or even activated by the activity of phospholipase C (PLC) (Montell et al., 2002). PLC activity is regulated externally by $\mathrm{G}$ protein coupled receptors (GPCRs). GPCRs in the cell membrane dissociate the $G$ protein into active $G \alpha$ and $G \beta \gamma$ when an agonist binds to it. PLC is activated and it hydrolyses phosphatidylinositol 4,5-bisphosphate producing two intracellular messengers: diacylglycerol and inositol 1,4,5-trisphosphate $(\operatorname{Ins}(1,4,5)$ P3). Diacylglycerol remains in the membrane and it is one of the candidates for the regulation/activation of TRPs (Hardie et al., 2002). Ins(1,4,5)P3 diffuses in the cytoplasm and increases intracellular $\mathrm{Ca}^{2+}$ by releasing it from the endoplasmic reticulum (Rhee and Bae, 1997). The mechanism of TRP channels beyond the activation of PLC is not described in detail here; a good review on this process can be found in Clapham (2003), Pedersen et al. (2005), Minke and Cook (2002) and Montell et al. (2002). Interestingly, PLC-dependent (such as TRPV1 and TRPV2) and -independent TRPs have been identified, but the physiological processes are still poorly understood. The regulation by the activity of PLC seems to account for increased sensitivity in inflammation (allodynia and hyperalgesia) among other regulatory processes acting on thermoreceptors (Davis et al., 2000; Montell et al., 2002; del Camino et al., 2010). The regulation explained above shows how TRPs are also regulated by internal, chemical and long-lasting signals.

\section{Neuronal level}

Similar to the aforementioned classification of the TRP channels, thermosensitive afferent neurons can be classified depending on the type of receptor they have. Thermoreceptors are found in different tissues; in the case of external temperature changes, they are situated in the free nerve endings of afferent neurons (Schepers and Ringkamp, 2010). In the innocuous range, there is an independent transduction and transmission of cold and hot stimuli information, since neurons with either cold or hot receptors have been found. Noxious cold and heat are mainly mediated by the same afferents, though there are some fibres activated specifically by just noxious cold or heat temperatures (Schepers and Ringkamp, 2010). Thus, there are primary afferents dedicated separately to the innocuous and noxious thermosensations. On the other hand, multimodal neurons (hot and cold transduction as well as the activation to other stimuli such as pressure, osmolality, etc.) have also been found, especially in the noxious range (LaMotte and Campbell, 1978; Campbell and LaMotte, 1983). Additionally, some mechanoreceptors are also sensitive to cold and heat, but their role in temperature sensation is not well understood (Schepers and Ringkamp, 2010). A consequence for these facts is that temperature sensitivity is not even around the body; instead the body shows varied sensitivities to temperature among different regions of the skin (Adair, 1999).

Heat is transferred from a heat reservoir to a cold one; thus a thermal gradient is created. Therefore, the temperature on the skin surface differs from the temperature inside. Additionally, human skin has three layers: epidermis, dermis and subcutaneous tissue. Thus, the heat is transferred through the tissue and a thermal gradient appears which stimulates the thermoreceptors differently depending on their location. Strikingly, innocuous cold and hot thermoreceptors are situated at a different depth in human skin. Cold receptors are found in the epidermis (Dhaka et al., 2008) at a depth of $0.2 \mathrm{~mm}$, whereas warm receptors are found deeper, approximately at a depth of $0.5 \mathrm{~mm}$ (Figure 2) (Lv and Liu, 2007). Noxious hot receptors (C fibres) have been found to be in a depth range of 0.2 $0.6 \mathrm{~mm}$ (Tillman et al., 1995). The depth of cold nociceptors might be the same, since TRPV1 and TRPA1 (remember that nociceptors are multimodal, the same afferent fibre receives and carries noxious cold and hot information) are

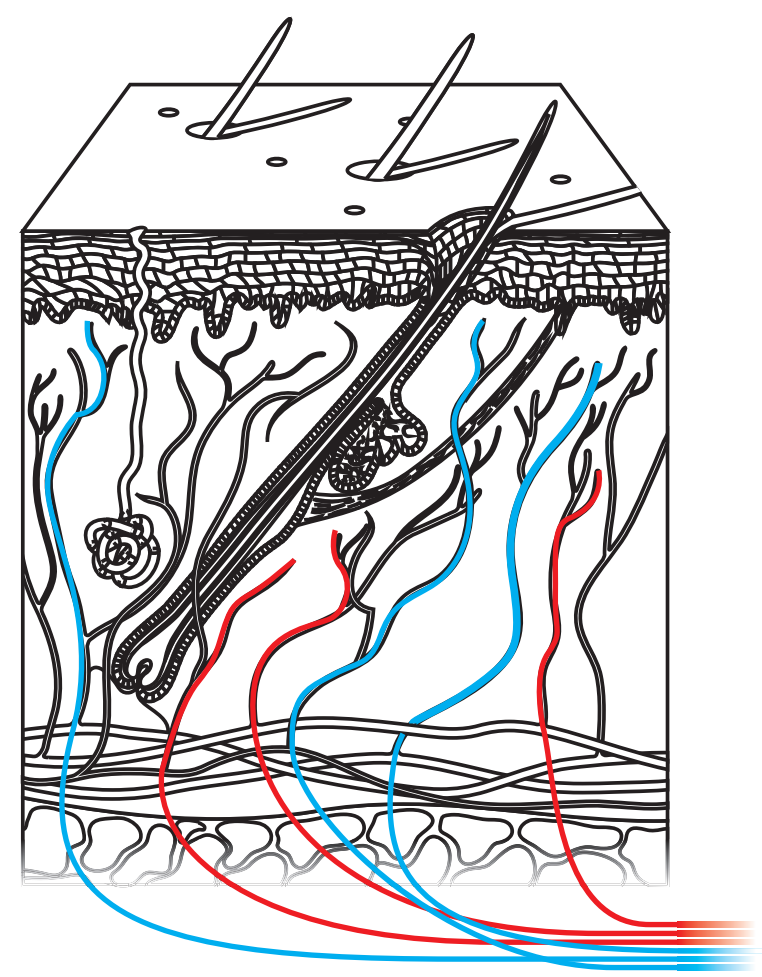

Figure 2: Diagram of the thermoreceptors in the skin. Note that on average hot receptors are found deeper in the skin. 
expressed mainly in the same neurons (Patapoutian et al., 2003). The fact that cold and hot receptors do not share the same depth has immediate consequences in the capacity to perceive stimuli by each type of receptor. Cold receptors temperature drops faster than warm receptor temperature; therefore their temperature is also increased more quickly. Consequently, the detection time of the warm receptors is slower than the cold receptors (Lv and Liu, 2007).

Another important characteristic is that each kind of thermosensitive afferent neurons is grouped into different types of fibres. Thus, warm (innocuous hot) sensitive fibres are mainly C fibres (Mackenzie et al., 1975; LaMotte and Campbell, 1978). These are unmyelinated and they have the smallest diameter, approximately $1-2 \mu \mathrm{m}$ (Lv and Liu, 2007), implying that they are the slowest conducting fibres. Cold sensation is mediated by $\mathrm{A} \delta$ and $\mathrm{C}$ fibres (Mackenzie et al., 1975; McKemy et al., 2002; Lv and Liu, 2007). A $\delta$ fibres are myelinated and have a diameter of around 3 $\mathrm{mm}$; hence they conduct faster than C fibres (Lv and Liu, 2007). Remarkably, this separation observed at the skin level of hot and cold information is kept in the spinal cord and even in some areas of the brain as it will be explained below (Craig and Dostrovsky, 2001; Craig, 2002, 2003). Besides, a feature that cold and hot sensitive fibres share is that they both branch at the end into 5-10 unmyelinated parts (Figure 2) (Adair, 1999).

Noxious cold and hot receptors are coupled to $\mathrm{C}$ and A $\delta$ fibres (Campbell and LaMotte, 1983; Caterina et al., 1997; Clapham et al., 2001). There is evidence that demonstrates that both fibres are involved in noxious temperature perception. In noxious hot sensation, $A \delta$ fibres conduct and make us feel the first fast pain onset, while $\mathrm{C}$ fibres firing explains the second pain sensation that lasts longer. Some researchers have blocked $\mathrm{A} \delta$ and they have observed the disappearance of the first fast pain (Price et al., 1977; Campbell and LaMotte, 1983). Importantly, the activity of the fibres correlates with the psychological pain perception of the stimuli (Schepers and Ringkamp, 2010). Interestingly, blocking $A \delta$ fibres in the presence of a cold stimulus results in burning or heat experience (Mackenzie et al., 1975; Craig and Bushnell, 1994). This observation is the same phenomenon noted in the thermal grill illusion (Yarnitsky and Ochoa, 1990); it suggests that hot and cold information input is already modulated at the spinal cord by cold afferents and that sensation depends on the integration of various inputs. Additionally, two different types of A $\delta$ fibre nociceptors have been identified depending on their response to heat stimuli. Type I fibres have a high temperature threshold, around $53^{\circ} \mathrm{C}$, and type II fibres have a lower activation point at approximately $47^{\circ} \mathrm{C}$ (Treede et al., 1995; Caterina et al., 1999; Schepers and Ringkamp, 2010).
The opening of the TRP channels leads to the polarization of the neuron, thus triggering the firing of a signal. Remarkably, the amplitude of the polarisation is the same for any stimuli in any neuron in the nervous system, meaning that information is not coded by the intensity of the polarisation. In fact, the intensity of the signal perceived is related to the frequency of the firing. In other words, the information is coded by the number of polarisation peaks per unit of time and not their amplitude. Thus, an abrupt change in temperature causes a high firing frequency that implies an intense signal. In particular, cold thermoreceptors increase their firing rate when temperature cools down and decrease their activity when temperatures rise, and vice versa for hot thermoreceptors (Kenshalo et al., 1968; Lv and Liu, 2007; Schepers and Ringkamp, 2010). Hence, during and after the temperature change, the activity of cold and hot sensors presents the biggest difference in firing rate. After the transient increase (or decrease) in firing rate, the neuron comes back to a steady level; this means thermoreceptors adapt to long-lasting stimuli (Hensel and Zotterman, 1951). Additionally, the peak impulse frequency of the cold receptors lasts longer than that of the warm thermoreceptors (Lv and Liu, 2007). This behaviour of the thermoreceptors at the skin level has direct effects on our perception of temperature.

To sum up, temperature sensation has dedicated specific fibres for each temperature range. It should be noted that a neuron is capable of reacting to both cold and hot stimuli due to the presence of cold and hot channels in its membrane (McKemy et al., 2002). Only C fibres are involved in innocuous warm stimuli sensation and both $\mathrm{C}$ and $\mathrm{A} \delta$ fibres in innocuous cold stimuli detection. Noxious stimuli are perceived by multimodal $\mathrm{C}$ and $\mathrm{A} \delta$ fibres. It is the integration and modulation of both types of fibres that leads to a correct sensation of noxious temperature. These differences in the type of fibres imply that thermal information has different speed and signal timing for each thermal range. Nevertheless, the final sensation of temperature arises from the complex interaction of the overlapping activity of different population of neurons. Accounts of this complex synergy are found in some bizarre sensory phenomena: paradoxical heat and cold, thermal grill illusion and cold allodynia (Craig and Bushnell, 1994; Craig et al., 1996; Craig, 2002).

\section{Central nervous system level}

Temperature and pain information goes through the spinothalamic tract. This tract is composed of afferent 
neurons that synapse in the substantia gelatinosa of the dorsal horn and the second-order neurons that decussate and ascend in the opposite tract (Figure 3) (Patapoutian et al., 2003). Research studies have revealed a labelled line of temperature information (as well as for other somatosensory information) from the skin to the brain, showing that there are dedicated pathways for each kind of information (Figure 4) (Craig, 2003).

Innocuous and noxious temperature-sensitive neurons synapse in the superficial laminae, lamina I and lamina II, of the dorsal horn (Caterina et al., 1999; Clapham et al., 2001; Patapoutian et al., 2003). Lamina I contains three different types of cells according to their morphology: fusiform, pyramidal and multipolar (Han et al., 1998). Fusiform cells respond to noxious hot stimuli and to pinch; this is supported by the fact that they seem to have unmyelinated axons (as aforementioned, noxious stimuli are perceived by $\mathrm{C}$ fibres). Pyramidal cells are responsive to innocuous cold and multipolar cells have been identified to be polymodal (heat, pinch and cold) and as nociceptive-specific (Zhang et al., 1996). These three different morphologies coincide with three distinct physiologies: nociceptivespecific, thermoreceptive-specific and polymodal nociceptive (Price et al., 1978; Zhang et al., 1996). Evidence that supports a labelled line of temperature sensation is the distribution of the channels. TRPV1 has been observed in the superficial laminae, laminae I and II (Caterina et al., 1999; Clapham et al., 2001). TRPV2 is mainly expressed in the superficial laminae, and TRPV3 and TRPV4 have been found in lamina I (Andrew and Craig, 2001; Patapoutian et al., 2003). Furthermore, the hypothalamus and the brainstem send projections to these laminae, indicating that there is already a modification and modulation of the signal at this stage (Holstege, 1988).

Strikingly, evidence of labelled-line logic in the brain has been found in the Drosophila brain. Gallio et al. (2011) showed a clear segregation of the cold and hot information-carrying neurons. Cold-sensing neurons target a glomerulus at the lateral margin of the proximal antennal protocerebrum (PAP), whereas hot-sensing neurons project to a separated glomerulus in the same area. It is important to highlight that they do not overlap. This arrangement creates a thermotopic map in the fly's PAP that translates to a functional representation of temperature in the brain. Furthermore, it is noteworthy that the anatomical independence of each temperature sensing neuron leads also to a clear behaviour consequence. Flies that had either the cold- or hot-sensing neurons silenced exhibited a selective deficit of avoidance to cold and hot stimuli when cold or hot glomerulus were inactivated, respectively (Gallio et al., 2011).

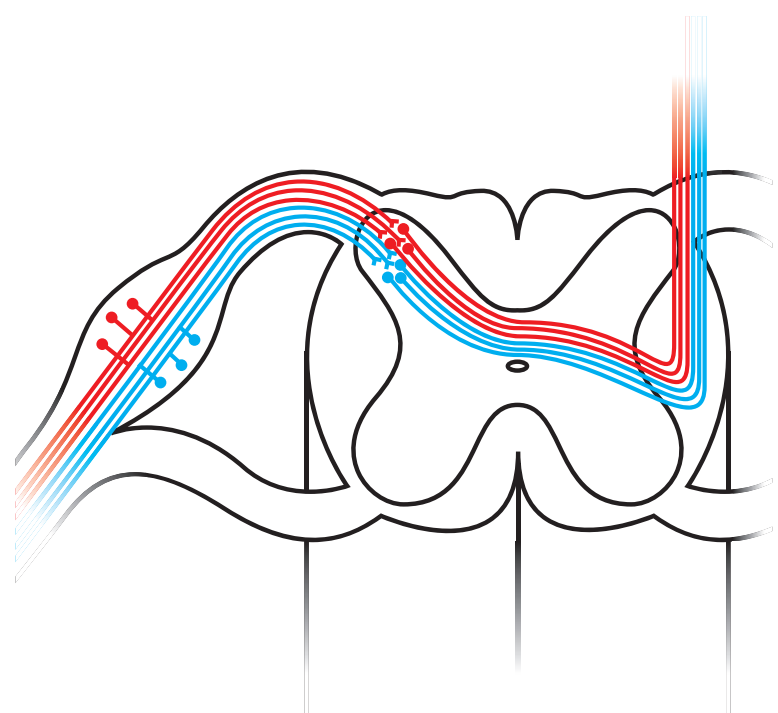

Figure 3: Diagram of primary afferent neurons carrying hot and cold temperature information to the spinal cord (Caterina et al., 1999; Clapham et al., 2001; Patapoutian et al., 2003).

Note that hot and cold innocuous information travels in separated fibres (represented in the figure), whereas hot and cold noxious information can be transmitted in the same primary afferent (not represented here for clarity).

Recently, a study conducted by Frank et al. (2015) found the high brain areas where thermosensory information converge: the mushroom body, the lateral horn and the posterior lateral protocerebrum. Most of the neurons that innervate the hot and cold glomeruli already target both types of cells, so they represent the first point of convergence of hot and cold temperature. This research group also identified fast- and slow-adapting neurons; these are projections from the PAP to the areas aforementioned activated by either hot or cold stimuli. Fast-adapting neurons are thought to convey temporal information (start and end). Slow-adapting neurons may encode for the magnitude of a temperature change (Frank et al., 2015). These findings are promising and the authors suggest a similar mechanism in mammals (Gallio et al., 2011).

Some insights have been achieved in the brain areas of humans and primates that become more active with temperature changes and information. Temperature neurons ascending via the spinothalamic tract send axons to the posterior part of the ventromedial nucleus (Lenz et al., 1993; Davis et al., 1999). Contrary to what would be expected, temperature information does not target the parietal somatosensory cortex. Innocuous temperature elicits activity in the right anterior insula and the orbitofrontal cortex (Craig et al., 2000) and 


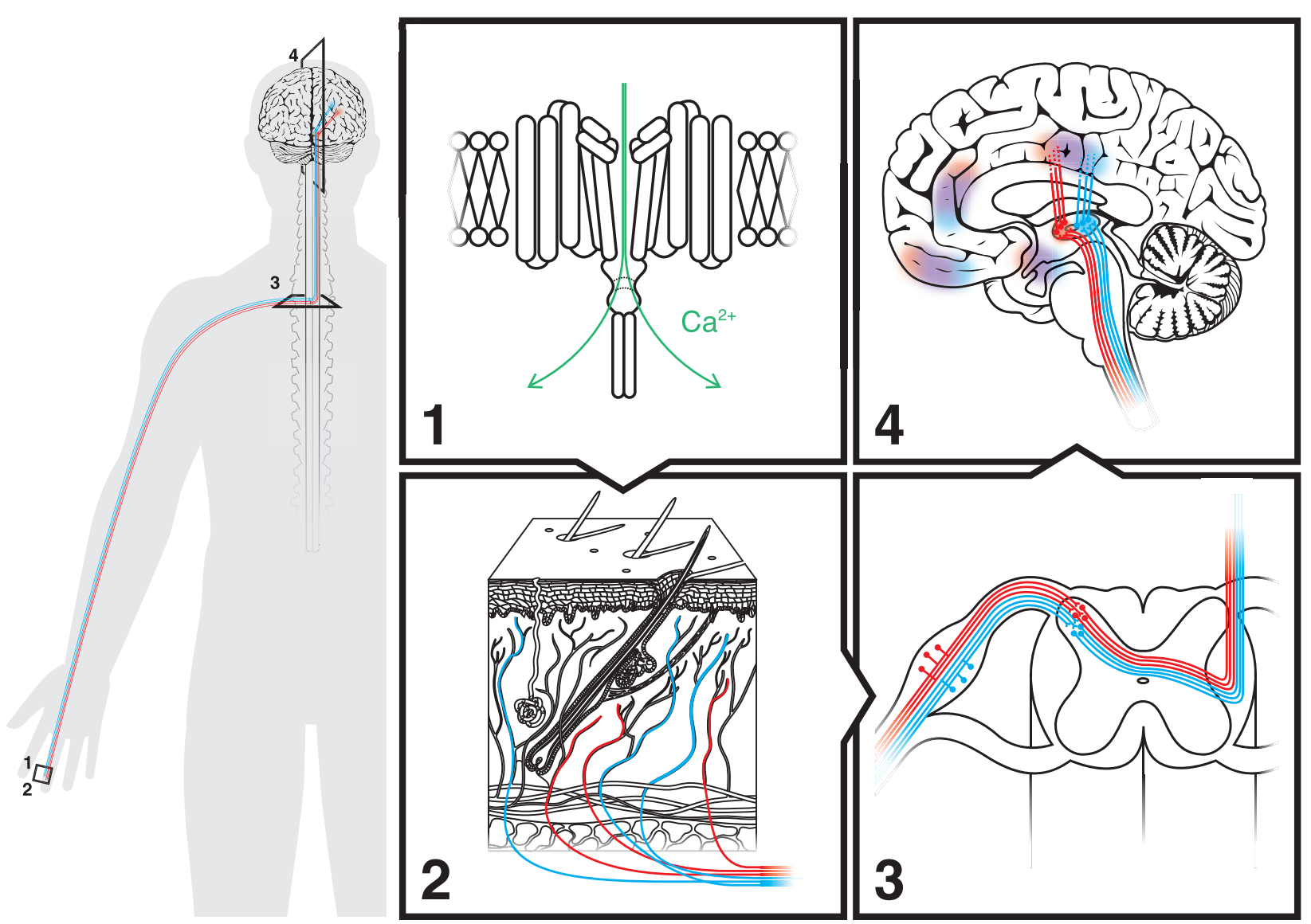

Figure 4: Diagram of the 'highway' to thermosensation, from the proteins to the brain.

noxious thermal stimuli activates the anterior cingulate cortex and the dorsal posterior insula (Figure 5) (Craig et al., 1996; Brooks et al., 2002). The journey that temperature information makes from the thermoreceptors to the brain is not futile; the messages are used by the hypothalamus, integrator of thermal internal and external information and responsible of thermogenesis (Boulant, 1998; Nagashima et al., 2000). The hypothalamus is located under the thalamus and it is the main chief in the hormone regulation (Figure 5). It has also been described to be the centre of control of emotions and other behavioural functions. Interestingly, temperature among other bodily feelings has an inherent association with emotion; it has a motivational aspect. It is obvious that temperature perception has a homeostatic role and it is related to the body's needs and survival (Mower, 1976; Satinoff, 1978). The feelings that arise with thermoperception are directly related to homeostasis and behavioural motivations of survival (Craig, 2002). It is probable that the synergic integration and interaction between the homeostatic centres in the brainstem and hypothalamus with the thermal representation in the insular, anterior cingulate and orbitofrontal cortex result in the feeling, awareness and perception of temperature (Craig, 2002).

The main area involved in thermoregulation is the preoptic-anterior hypothalamus (Boulant, 1980). The posterior nucleus of the hypothalamus, more concretely the dorsomedial region, seems to have an important role in cold responses such as shivering (Stuart et al., 1961; Halvorson and Thornhill, 1993; Nagashima et al., 2000). These areas elicit a variety of responses to control corporal temperature. When the individual's temperature decreases, the body carries out heat retention responses such as shivering, cutaneous vasoconstriction and behavioural responses. When body temperature increases, the body undergoes heat loss responses such as panting, sweating and vasodilatation. Along with the idea that TRP channels are expressed in the central nervous system, these types of responses are also provoked when some preoptic temperature-sensitive neurons are warmed or cooled down (Boulant, 1998). Nevertheless, a major proportion of the neurons found in this area are temperature insensitive. 


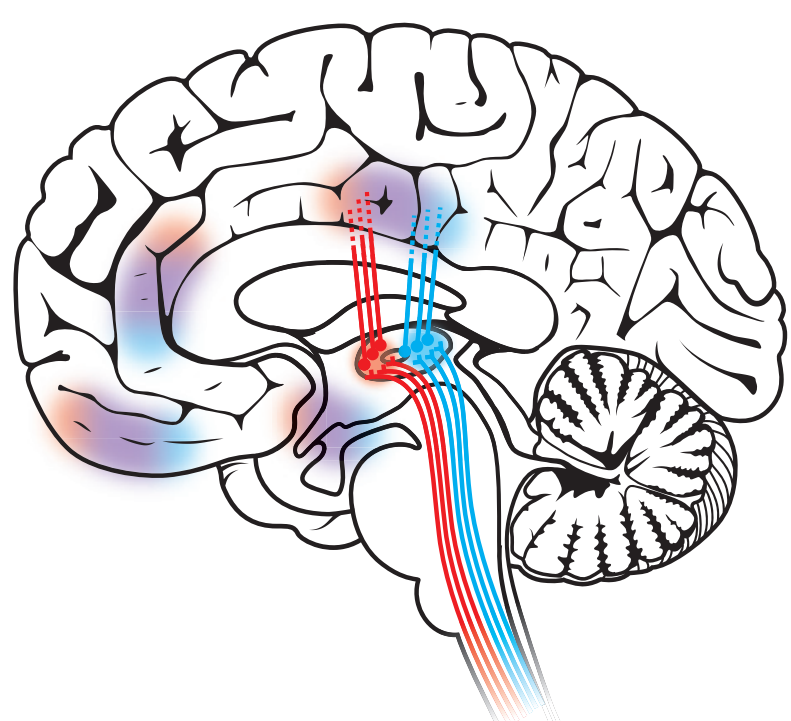

Figure 5: Diagram of the main areas of the human brain active with temperature information and changes (insular cortex, anterior cingulate cortex, orbitofrontal cortex and hypothalamus). Connections from the thalamus to each area have not been drawn for clarity reasons. The insular cortex cannot be seen in this sagittal plane; hence the dashed lines represent the connections to this area. Modified from Craig (2002).

Similar to the distinction between cells dedicated to warm and cold information in the Drosophila brain, warm- and cold-sensitive neurons in the preoptic area have been seen to increase their firing rate to external (skin) and direct (preoptic) warming and cooling, respectively (Boulant and Hardy, 1974; Boulant and Gonzalez, 1977). Interestingly, warm-sensitive neurons seem to be the major contributor in the integration of the information (Boulant, 1998) and the regulation of temperature (Zhang et al., 1995; Chen et al., 1998). Warm-sensitive neurons send dendrites to at least two information sources: perpendicular and medially to the third ventricle (Boulant, 1998). It has also been found that these neurons contribute more to the control of shivering, non-shivering and vasomotion (Zhang et al., 1995; Chen et al., 1998).

\section{Concluding remarks}

Thermoperception is conditioned by the way information transduces and transmits; it is not as simple as if we had thermometers. We have at least six TRP channels (TRPV1, TRPV2, TRPV3, TRPV4, TRPM8 and TRPA1) that respond nonlinearly to temperature changes. Furthermore, they are expressed in different groups of neurons that have different characteristics; this information goes at different speeds, through different pathways and arrives to the brain separately (Figure 4). Interestingly, it seems that the body has evolved to group pain and noxious stimuli separated from precise and more accurate innocuous stimuli.

Thermoperception is also highly dependent by the way each element communicates with one another. As it is revealed in the thermal grill illusion and the paradoxical burning sensation, there are excitatory and inhibitory interactions between different subsets of neurons. The arrangement of the elements implicated in thermosensation is also determined for the resulting sensation. For instance, differences in the distribution of the expression of TRP channels have been found across different species. Consequently, each living creature generates its own thermal map that determines its relation and reaction to the environment's stimuli (Caterina et al., 1999; Hori et al., 1999; Guler et al., 2002; Peier et al., 2002; Smith et al., 2002; Xu et al., 2002). Therefore, distinct species generate a different perception of the world, not just because their nervous systems are different, but because their entire biology has a particular structure and arrangement.

The way we perceive the world seems to be determined by the physiological structure of each element involved in thermosensation. Nevertheless, to completely comprehend temperature perception, each distinguished mechanism should be integrated under a holistic perspective. We would understand how we distinguish a cold object from a hot one, how we perceive ambient temperature and how this affects our body, which is the body response, etc. However, to achieve this, all the thermodynamic variables of the organism need to be integrated in the analysis, such as the way we generate and maintain internal heat, how heat transmits and the intrinsic thermal conductivity of the skin, how the thermal gradient is created in our skin and body, etc. (Torvi and Dale, 1994; Lv and Liu, 2007).

This review is focused only on the characteristics of the thermoperception mechanisms. Interestingly, some similarities with some exceptions are pinpointed across all the senses. For example, metabotropic receptors (GPCRs) are used to transduce the stimuli in vision, taste, pain, etc. Strikingly, the proteins that configure these receptors belong to the same family, TRP channels (Patapoutian et al., 2003; Zhang et al., 2003; Pedersen et al., 2005). The first TRP channel identified was actually one involved in fly's vision (Cosens and Manning, 1969). It is noteworthy that the labelled-line hypothesis (Figure 4) is supported as far as it is known by all the senses; even the eye shows different pathways for different channels of the visual information. 
Understanding the mechanism of thermosensation would obviously lead us to interpret different body responses such as fever and heatstroke or how the body encounters changes in the ambient temperature. But we would also find direct applications in quotidian life. The textile, air conditioning and heating industries could take advantage of the findings and they could apply them to improve all their products, looking for achieving and maintaining the temperature-comfort zone.

On the other hand, the comprehension of thermoperception would even help us to elucidate the creation of concepts of heat and temperature. This could be applied by teachers to optimise the teaching of these thermodynamic terms. Although there are still many gaps to fill, we are certainly on the highway to thermosensation.

Acknowledgments: We would like to thank Karishma Mahtini for her accurate comments and for proofreading the paper and to Mario Martinez Cepa for his altruistic and artistic drawings.

\section{References}

Adair, R.K. (1999). A model of the detection of warmth and cold by cutaneous sensors through effects on voltage-gated membrane channels. Proc. Nat. Acad. Sci. USA 96, 11825-11829.

Andrew, D. and Craig, A. (2001). Spinothalamic lamina I neurones selectively responsive to cutaneous warming in cats. J. Physiol. 537, 489-495.

Arrigoni, C., Rohaim, A., Shaya, D., Findeisen, F., Stein, R.A., Nurva, S.R.,... Minor, D.L. (2016). Unfolding of a temperature-sensitive domain controls voltage-gated channel activation. Cell 164, 922-936.

Askwith, C.C., Benson, C.J., Welsh, M.J., and Snyder, P.M. (2001). $\mathrm{DEG} / \mathrm{ENaC}$ ion channels involved in sensory transduction are modulated by cold temperature. Proc. Nat. Acad. Sci. USA 98, 6459-6463.

Bagriantsev, S.N., Clark, K.A., and Minor, D.L., Jr. (2012). Metabolic and thermal stimuli control K(2P)2.1 (TREK-1) through modular sensory and gating domains. EMBO J. 31, 3297-3308.

Bautista, D.M., Siemens, J., Glazer, J.M., Tsuruda, P.R., Basbaum, A.I., Stucky, C.L.,... Julius, D. (2007). The menthol receptor TRPM8 is the principal detector of environmental cold. Nature 448, 204-208.

Bear, M.F., Connors, B.W., and Paradiso, M.A. (2007). Neuroscienze. esplorando il cervello. With CD-ROM Elsevier srl.

Benham, C.D., Gunthorpe, M.J., and Davis, J.B. (2003). TRPV channels as temperature sensors. Cell Calcium 33, 479-487.

Boulant, J. (1980). Hypothalamic control of thermoregulation: neurophysiological basis. Handbook of the Hypothalamus 3, $1-82$.

Boulant, J. (1998). Hypothalamic neurons: mechanisms of sensitivity to temperature. Ann. NY Acad. Sci. 856, 108-115.
Boulant, J.A. (2000). Role of the preoptic-anterior hypothalamus in thermoregulation and fever. Clin. Infect. Dis. 31, S157-S161.

Boulant, J.A. and Dean, J.B. (1986). Temperature receptors in the central nervous system. Annu. Rev. Physiol. 48, 639-654.

Boulant, J.A. and Gonzalez, R.R. (1977). The effect of skin temperature on the hypothalamic control of heat loss and heat production. Brain Res. 120, 367-372.

Boulant, J.A. and Hardy, J.D. (1974). The effect of spinal and skin temperatures on the firing rate and thermosensitivity of preoptic neurones. J. Physiol. 240, 639-660.

Brauchi, S., Orio, P., and Latorre, R. (2004). Clues to understanding cold sensation: thermodynamics and electrophysiological analysis of the cold receptor TRPM8. Proc. Nat. Acad. Sci. USA 101, 15494-15499.

Brauchi, S., Orta, G., Salazar, M., Rosenmann, E. and Latorre, R. (2006). A hot-sensing cold receptor: C-terminal domain determines thermosensation in transient receptor potential channels. J. Neurosci. 26, 4835-4840.

Brooks, J.C., Nurmikko, T.J., Bimson, W.E., Singh, K.D., and Roberts, N. (2002). fMRI of thermal pain: effects of stimulus laterality and attention. Neurolmage 15, 293-301.

Campbell, J.N. and LaMotte, R.H. (1983). Latency to detection of first pain. Brain Res. 266, 203-208.

Carpenter, D.O. (1967). Temperature effects on pacemaker generation, membrane potential, and critical firing threshold in aplysia neurons. J. Gen. Physiol. 50, 1469-1484.

Caterina, M.J., Schumacher, M.A., Tominaga, M., Rosen, T.A., Levine, J.D., and Julius, D. (1997). The capsaicin receptor: a heat-activated ion channel in the pain pathway. Nature 389, 816-824.

Caterina, M.J., Rosen, T.A., Tominaga, M., Brake, A.J., and Julius, D. (1999). A capsaicin-receptor homologue with a high threshold for noxious heat. Nature 398, 436-441.

Chen, X., Hosono, T., Yoda, T., Fukuda, Y., and Kanosue, K. (1998). Efferent projection from the preoptic area for the control of non-shivering thermogenesis in rats. J. Physiol. 512, 883-892.

Chowdhury, S., Jarecki, B.W., and Chanda, B. (2014). A molecular framework for temperature-dependent gating of ion channels. Cell 158, 1148-1158.

Chung, M.K., Guler, A.D., and Caterina, M.J. (2005). Biphasic currents evoked by chemical or thermal activation of the heatgated ion channel, TRPV3. J. Biol. Chem. 280, 15928-15941.

Clapham, D.E. (2003). TRP channels as cellular sensors. Nature 426, 517-524.

Clapham, D.E. and Miller, C. (2011). A thermodynamic framework for understanding temperature sensing by transient receptor potential (TRP) channels. Proc. Nat. Acad. Sci. USA 108, 19492-19497.

Clapham, D.E., Runnels, L.W., and Strübing, C. (2001). The TRP ion channel family. Nat. Rev. Neurosci. 2, 387-396.

Cosens, D., and Manning, A. (1969). Abnormal electroretinogram from a Drosophila mutant. Nature 224, 285-287.

Craig, A.D. (2002). How do you feel? Interoception: the sense of the physiological condition of the body. Nat. Rev. Neurosci. 3, 655-666.

Craig, A. (2003). A new view of pain as a homeostatic emotion. Trends Neurosci. 26, 303-307.

Craig, A.D. and Bushnell, M.C. (1994). The thermal grill illusion: unmasking the burn of cold pain. Science 265, 252-255.

Craig, A.D. and Dostrovsky, J.O. (2001). Differential projections of thermoreceptive and nociceptive lamina I trigeminothalamic 
and spinothalamic neurons in the cat. J. Neurophysiol. 86 , 856-870.

Craig, A., Reiman, E., Evans, A., and Bushnell, M. (1996). Functional imaging of an illusion of pain. Nature 384, 258-260.

Craig, A.D., Chen, K., Bandy, D., and Reiman, E.M. (2000). Thermosensory activation of insular cortex. Nat. Neurosci. 3, 184-190.

Davis, K.D. and Pope, G.E. (2002). Noxious cold evokes multiple sensations with distinct time courses. Pain 98, 179-185.

Davis, K.D., Lozano, R.M., Manduch, M., Tasker, R.R., Kiss, Z.H., and Dostrovsky, J.O. (1999). Thalamic relay site for cold perception in humans. J. Neurophysiol. 81, 1970-1973.

Davis, J.B., Gray, J., Gunthorpe, M.J., Hatcher, J.P., Davey, P.T., Overend, P., Harries MH, Latcham J, Clapham C, Atkinson, K., et al. (2000). Vanilloid receptor-1 is essential for inflammatory thermal hyperalgesia. Nature 405, 183-187.

del Camino, D., Murphy, S., Heiry, M., Barrett, L.B., Earley, T.J., Cook, C.A., Petrus, MJ., Zhao, M., D’Amours, M., Deering, N., et al. (2010). TRPA1 contributes to cold hypersensitivity. J. Neurosci. 30, 15165-15174.

Dhaka, A., Earley, T.J., Watson, J., and Patapoutian, A. (2008). Visualizing cold spots: TRPM8-expressing sensory neurons and their projections. J. Neurosci. 28, 566-575.

Frank, D.D., Jouandet, G.C., Kearney, P.J., Macpherson, L.J., and Gallio, M. (2015). Temperature representation in the drosophila brain. Nature 519, 358-361.

Gallio, M., Ofstad, T.A., Macpherson, L.J., Wang, J.W., and Zuker, C.S. (2011). The coding of temperature in the drosophila brain. Cell 144, 614-624.

Grandl, J., Hu, H., Bandell, M., Bursulaya, B., Schmidt, M., Petrus, M., and Patapoutian, A. (2008). Pore region of TRPV3 ion channel is specifically required for heat activation. Nat. Neurosci. 11, 1007-1013.

Guler, A.D., Lee, H., lida, T., Shimizu, I., Tominaga, M., and Caterina, M. (2002). Heat-evoked activation of the ion channel, TRPV4. J. Neurosci. 22, 6408-6414.

Halvorson, I. and Thornhill, J. (1993). Posterior hypothalamic stimulation of anesthetized normothermic and hypothermic rats evokes shivering thermogenesis. Brain Res. 610, 208-215.

Han, Z., Zhang, E., and Craig, A. (1998). Nociceptive and thermoreceptive lamina I neurons are anatomically distinct. Nat. Neurosci. 1, 218-225.

Hardie, R.C., Martin, F., Cochrane, G., Juusola, M., Georgiev, P., and Raghu, P. (2002). Molecular basis of amplification in drosophila phototransduction: roles for $\mathrm{G}$ protein, phospholipase C, and diacylglycerol kinase. Neuron 36, 689-701.

Hensel, H. and Zotterman, Y. (1951). The response of the cold receptors to constant cooling. Acta Physiol. Scand. 22, 96-105.

Hille, B. (2001). Ion Channels of Excitable Membranes (Sunderland, $M A:$ Sinauer).

Holstege, G. (1988). Direct and indirect pathways to lamina I in the medulla oblongata and spinal cord of the cat. Progr. Brain Res. 77, 47-94.

Hori, A., Minato, K., and Kobayashi, S. (1999). Warming-activated channels of warm-sensitive neurons in rat hypothalamic slices. Neurosci. Lett. 275, 93-96.

Irie, K., Shimomura, T., and Fujiyoshi, Y. (2012). The C-terminal helical bundle of the tetrameric prokaryotic sodium channel accelerates the inactivation rate. Nat. Commun. 3, 793.
Jiang, Y., Lee, A., Chen, J., Cadene, M., Chait, B.T., and MacKinnon, R. (2002). Crystal structure and mechanism of a calcium-gated potassium channel. Nature 417, 515-522.

Kenshalo, D.R., Holmes, C.E., and Wood, P.B. (1968). Warm and cool thresholds as a function of rate of stimulus temperature change. Percept. Psychophys. 3, 81-84.

LaMotte, R.H. and Campbell, J.N. (1978). Comparison of responses of warm and nociceptive C-fiber afferents in monkey with human judgments of thermal pain. J. Neurophysiol. 41, 509-528.

Lee, H.M., Cho, C.K., Yun, M.H., and Lee, M.W. (1998). Development of a temperature control procedure for a room air-conditioner using the concept of just noticeable difference (JND) in thermal sensation. Int. J. Ind. Ergonom. 22, 207-216.

Lenz, F.A., Seike, M., Richardson, R.T., Lin, Y.C., Baker, F.H., Khoja, I., Jaeger, C.J., Gracely, R.H. (1993). Thermal and pain sensations evoked by microstimulation in the area of human ventrocaudal nucleus. J. Neurophysiol. 70, 200-212.

Lv, Y. and Liu, J. (2007). Effect of transient temperature on thermoreceptor response and thermal sensation. Build. Environ. 42, 656-664.

Mackenzie, R.A., Burke, D., Skuse, N.F., and Lethlean, A.K. (1975). Fibre function and perception during cutaneous nerve block. J. Neurol. Neurosurg. 38, 865-873.

Maingret, F., Lauritzen, I., Patel, A.J., Heurteaux, C., Reyes, R., Lesage, F., Lazdunski, M., Honore, E. (2000). TREK-1 is a heatactivated background $\mathrm{K}^{+}$channel. EMBO J. 19, 2483-2491.

McKemy, D.D., Neuhausser, W.M., and Julius, D. (2002). Identification of a cold receptor reveals a general role for TRP channels in thermosensation. Nature 416, 52-58.

Mezey, É, Tóth, Z.E., Cortright, D.N., Arzubi, M.K., Krause, J.E., Elde, R., Guo, A., Blumberg, P.M., Szallasi, A. (2000). Distribution of mRNA for vanilloid receptor subtype 1 (VR1), and VR1-like immunoreactivity, in the central nervous system of the rat and human. Proc. Natl. Acad. Sci. USA 97, 3655-3660.

Minke, B. and Cook, B. (2002). TRP channel proteins and signal transduction. Physiol. Rev. 82, 429-472.

Mio, K., Mio, M., Arisaka, F., Sato, M., and Sato, C. (2010). The C-terminal coiled-coil of the bacterial voltage-gated sodium channel NaChBac is not essential for tetramer formation, but stabilizes subunit-to-subunit interactions. Prog. Biophys. Mol. Biol. 103, 111-121.

Montell, C., Birnbaumer, L., and Flockerzi, V. (2002). The TRP channels, a remarkably functional family. Cell 108, 595-598.

Mower, G.D. (1976). Perceived intensity of peripheral thermal stimuli is independent of internal body temperature. J. Comp. Physiol. 90, 1152.

Nagashima, K., Nakai, S., Tanaka, M., and Kanosue, K. (2000). Neuronal circuitries involved in thermoregulation. Auton. Neurosci. $85,18-25$.

Nagy, I. and Rang, H. (1999). Noxious heat activates all capsaicinsensitive and also a sub-population of capsaicin-insensitive dorsal root ganglion neurons. Neuroscience 88, 995-997.

Nilius, B., Talavera, K., Owsianik, G., Prenen, J., Droogmans, G., and Voets, T. (2005). Gating of TRP channels: a voltage connection? J. Physiol. 567, 35-44.

Patapoutian, A., Peier, A.M., Story, G.M., and Viswanath, V. (2003). ThermoTRP channels and beyond: mechanisms of temperature sensation. Nat. Rev. Neurosci. 4, 529-539. 
Payandeh, J. and Minor, D.L. (2015). Bacterial voltage-gated sodium channels (BacNaVs) from the soil, sea, and salt lakes enlighten molecular mechanisms of electrical signaling and pharmacology in the brain and heart. J. Mol. Biol. 427, 3-30.

Pedersen, S.F., Owsianik, G., and Nilius, B. (2005). TRP channels: an overview. Cell Calcium 38, 233-252.

Peier, A.M., Reeve, A.J., Andersson, D.A., Moqrich, A., Earley, T.J., Hergarden, A.C., Story, G.M., Colley, S., Hogenesch, J.B., McIntyre, P., et al. (2002). A heat-sensitive TRP channel expressed in keratinocytes. Science 296, 2046-2049.

Pierau, F., Torrey, P., and Carpenter, D.O. (1974). Mammalian cold receptor afferents: role of an electrogenic sodium pump in sensory transduction. Brain Res. 73, 156-160.

Pioletti, M., Findeisen, F., Hura, G.L., and Minor, D.L. (2006). Threedimensional structure of the KChIP1-Kv4. 3 T1 complex reveals a cross-shaped octamer. Nat. Struct. Mol. Biol. 13, 987-995.

Powl, A.M., O’Reilly, A.O., Miles, A.J., and Wallace, B.A. (2010). Synchrotron radiation circular dichroism spectroscopy-defined structure of the C-terminal domain of $\mathrm{NaChBac}$ and its role in channel assembly. Proc. Nat. Acad. Sci. USA 107, 14064-14069.

Price, D.D., Hu, J.W., Dubner, R., and Gracely, R.H. (1977). Peripheral suppression of first pain and central summation of second pain evoked by noxious heat pulses. Pain 3, 57-68.

Price, D.D., Hayes, R.L., Ruda, M., and Dubner, R. (1978). Spatial and temporal transformations of input to spinothalamic tract neurons and their relation to somatic sensations. J. Neurophysiol. 41, 933-947.

Rhee, S.G., and Bae, Y.S. (1997). Regulation of phosphoinositidespecific phospholipase C isozymes. J. Biol. Chem. 272, 15045-15048.

Satinoff, E. (1978). Neural organization and evolution of thermal regulation in mammals. Science 201, 16-22.

Schepers, R.J. and Ringkamp, M. (2010). Thermoreceptors and thermosensitive afferents. Neurosci. Biobehav. Rev. 34, 177-184.

Shaya, D., Findeisen, F., Abderemane-Ali, F., Arrigoni, C., Wong, S., Nurva, S.R., Loussouarn, G., Minor, D.L. (2014). Structure of a prokaryotic sodium channel pore reveals essential gating elements and an outer ion binding site common to eukaryotic channels. J. Mol. Biol. 426, 467-483.

Smith, G., Gunthorpe, M., Kelsell, R., Hayes, P., Reilly, P., Facer, P., Wright, J.E., Jerman, J.C., Walhin, J.P., Ooi, L. (2002). TRPV3 is a temperature-sensitive vanilloid receptor-like protein. Nature 418, 186-190.

Souslova, V., Cesare, P., Ding, Y., Akopian, A.N., Stanfa, L., Suzuki, R., Carpenter, K., Dickenson, A., Boyce, S., Hill, R., et al. (2000). Warm-coding deficits and aberrant inflammatory pain in mice lacking P2X 3 receptors. Nature 407, 1015-1017.

Story, G.M., Peier, A.M., Reeve, A.J., Eid, S.R., Mosbacher, J., Hricik, T.R., McIntyre, P., Jegla, T., Bevan, S., Patapoutian, A., et al. (2003). ANKTM1, a TRP-like channel expressed in nociceptive neurons, is activated by cold temperatures. Cell 112, 819-829.

Stuart, D., Kawamura, Y., and Hemingway, A. (1961). Activation and suppression of shivering during septal and hypothalamic stimulation. Exp. Neurol. 4, 485-506.

Tillman, D., Treede, R., Meyer, R.A., and Campbell, J.N. (1995). Response of $\mathrm{C}$ fibre nociceptors in the anaesthetized monkey to heat stimuli: estimates of receptor depth and threshold. J. Physiol. 485, 753-765.

Torvi, D. and Dale, J. (1994). A finite element model of skin subjected to a flash fire. J. Biomech. Eng 116, 250-255.
Treede, R., Meyer, R., Raja, S., and Campbell, J. (1995). Evidence for two different heat transduction mechanisms in nociceptive primary afferents innervating monkey skin. J. Physiol. 483, 747-758.

Voets, T., Droogmans, G., Wissenbach, U., Janssens, A., Flockerzi, V., and Nilius, B. (2004). The principle of temperature-dependent gating in cold-and heat-sensitive TRP channels. Nature 430, 748-754.

Voets, T., Talavera, K., Owsianik, G., and Nilius, B. (2005). Sensing with TRP channels. Nat. Chem. Biol. 1, 85-92.

Vriens, J., Nilius, B., and Voets, T. (2014). Peripheral thermosensation in mammals. Nat. Rev. Neurosci. 15, 573-589.

Watanabe, H., Vriens, J., Suh, S. H., Benham, C.D., Droogmans, G., and Nilius, B. (2002). Heat-evoked activation of TRPV4 channels in a HEK293 cell expression system and in native mouse aorta endothelial cells. J. Biol. Chem. 277, 47044-47051.

Xu, H., Ramsey, I.S., Kotecha, S.A., Moran, M.M., Chong, J.A., Lawson, D., Ge, P., Lilly, J., Silos-Santiago, I., Xie, Y., et al. (2002). TRPV3 is a calcium-permeable temperature-sensitive cation channel. Nature 418, 181-186.

Yarnitsky, D. and Ochoa, J.L. (1990). Release of cold-induced burning pain by block of cold-specific afferent input. Brain 113, 893-902.

Yu, F.H., Yarov-Yarovoy, V., Gutman, G.A., and Catterall, W.A. (2005). Overview of molecular relationships in the voltage-gated ion channel superfamily. Pharmacol. Rev. 57, 387-395.

Yuan, P., Leonetti, M.D., Pico, A.R., Hsiung, Y., and MacKinnon, R. (2010). Structure of the human BK channel $\mathrm{Ca}^{2+}$-activation apparatus at 3.0 ̊̊ resolution. Science 329, 182-186.

Zagotta, W.N., Olivier, N.B., Black, K.D., Young, E.C., Olson, R., and Gouaux, E. (2003). Structural basis for modulation and agonist specificity of HCN pacemaker channels. Nature 425, 200-205.

Zhang, Y., Yanase-Fujiwara, M., Hosono, T., and Kanosue, K. (1995). Warm and cold signals from the preoptic area: which contribute more to the control of shivering in rats? J. Physiol. 485, 195-202.

Zhang, E., Han, Z., and Craig, A. (1996). Morphological classes of spinothalamic lamina I neurons in the cat. J. Comp. Neurol. 367, 537-549.

Zhang, Y., Hoon, M.A., Chandrashekar, J., Mueller, K.L., Cook, B., Wu, D., Zuker, C.S., Ryba, N.J. (2003). Coding of sweet, bitter, and umami tastes: different receptor cells sharing similar signaling pathways. Cell 112, 293-301.

\section{Bionotes}

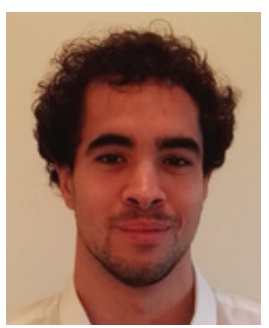

\section{Ivan Ezquerra-Romano}

Department of Cognitive, Perception and Brain Science, University College London, Gower Street, London WC1E 6BT, UK, ivan.romano.14@ucl.ac.uk

Ivan Ezquerra-Romano is an MSc Neuroscience undergraduate at University College London. His scientific interest is in consciousness, perception and neuropharmacology. He was awarded in the 
summer of 2016 with the Wellcome Trust Vacation Scholarship for doing research at his university in decision-making. He is also co-founder of a drugs harm reduction website (Drugsand.me) that provides reliable and evidence-based information for drug users.

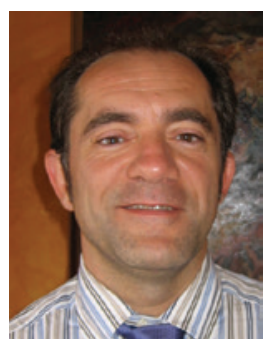

Angel Ezquerra,

Department of Didactics of Experimental

Science, Faculty of Education, Complutense

University of Madrid, C/Rector Royo

Villanova, s/n, E-28040 Madrid, Spain,

angel.ezquerra@edu.ucm.es.

http://orcid.org/0000-0002-5736-9867

Angel Ezquerra is a professor of Science Education in the Department of Didactics of Experimental Sciences, Complutense University of Madrid, Spain. His research is focused on the study of the inception of misconceptions in the understanding of physics. He has also carried out research on pedagogical content knowledge in science and on the strategies of teacher education. 